\title{
New structures simultaneously harboring class 1 integron and ISCR1-linked resistance genes in multidrug-resistant Gram-negative bacteria
}

\author{
Cancan Cheng ${ }^{\dagger}$, Jingjing Sun ${ }^{\dagger}$, Fen Zheng, Wenting Lu, Qiu Yang and Yongyu Rui ${ }^{*}$
}

\begin{abstract}
Background: The connection structure of class 1 integron and insertion sequence common region 1 (ISCR1) is called "complex class 1 integrons" or "complex sul1-type integrons", which is also known to be associated with many resistance genes. This structure is a powerful gene-capturing tool kit that can mobilize antibiotic resistance genes. In order to look for and study the structure among clinical multidrug-resistant (MDR) Gram-negative isolates, 63 isolates simultaneously harbored class 1 integron and ISCR1-linked resistance genes were isolated from 2309 clinical non-redundant MDR Gram-negative isolates in Nanfang Hospital in 2008-2013. The connecting regions between the class 1 integrons and ISCR1 were examined using PCR and DNA sequencing to determine the structures in these isolates.
\end{abstract}

Result: The two elements (the variable regions of the class 1 integron structures and the ISCR1-linked resistance genes) are connected in series among 63 isolates according to long-extension PCR and DNA sequencing. According to the kinds and permutations of resistance genes in the structure, 12 distinct types were identified, including 8 types that have never been described in any species. Several types of these structures are similar with the structures of other reports, but not entirely same.

Conclusion: This study is the first to determine the structure simultaneously harboring class 1 integron and ISCR1linked resistance genes by detecting the region connecting class 1 integrons and ISCR1 in a large number of MDR bacteria. These structures carrying various resistance genes were closely associated with multidrug resistance bacteria in Southern China.

Keywords: Multidrug-resistant, Class 1 integron, ISCR1, Gram-negative bacteria

\section{Background}

The increasing use of antimicrobial agents to treat Gramnegative bacterial infections has led to an increase in antibiotic resistance. Consequently, formerly routine therapies for many infectious diseases caused by multidrug-resistant (MDR) Gram-negative bacteria are now compromised. MDR bacteria evolve relatively quickly because the main driving force is lateral gene transfer, which is facilitated by a wide range of mobile genetic elements. The majority of

\footnotetext{
* Correspondence: yongyuruigroup@sina.com

${ }^{\dagger}$ Equal contributors

Laboratory Medicine Center, Nanfang Hospital, Southern Medical University, Guangzhou 510515, China
}

these elements are integrons and transposons (including unit transposons and insertion sequences) [1]. Insertion sequences with common regions (ISCRs) are a type of insertion sequence.

two previous studies, a total of 1329 and 1447 multirug Gram-negative bacteria isolated in 2008-2009, was vestigated for an ISCR1 [2] and a class 1 integron [3] reclively. In this study, 2309 clinical non-redundant Gram-negative isolates were isolated between 2008 13 at Nanfang Hospital, a 2200-bed tertiary-leve which carry a physical linkage between class 1 integrons and ISCR1 were focused. The ISCR1 and class 1 integrons 
were characterized using PCR and DNA sequencing as the methods described previously $[2,3]$. Of these strains, 63 isolates simultaneously harbored class 1 integrons and ISCR1-linked resistance genes were selected. The results were shown on the Table 1 . Based on the results, the region connecting the ISCR 1 and the 3 '-CS of the integron and the overall structures were investigated.

This structure which is usually called "complex class 1 integrons" or "complex sul1-type integrons", [4] is the large genetic element in which different class 1 integrons is associated with ISCR1. These elements are known to be associated with many resistance genes, encoding resistance to chloramphenicol, trimethoprim, quinolone, and $\beta$-lactam, [5] and have two notable structures. Besides, this structure is a powerful gene-capturing tool kit that can mobilize antibiotic resistance genes. The most of their structure comprises a typical class 1 integron with a $5^{\prime}$ conserved segment $\left(5^{\prime}-\mathrm{CS}\right)$, a $3^{\prime}-\mathrm{CS}$, and an intervening variable region (VR1), followed by a copy of ISCR 1 and then by an ISCR1-linked resistance gene region (VR2), which accommodates a variety of resistance genes. This region is, in turn, followed by a repetition of the $3^{\prime}$ - CS $[6,7]$.

\section{Methods}

According to the previous study, sixty three isolates simultaneously harbored class 1 integrons and ISCR1-linked resistance genes were selected for further analysis of the connecting the ISCR1 and the 3'-CS of the integron.

According the hypothesis connecting structure (Fig. 1) between the ISCR1 and the 3'-CS of the integron, primers A and B were designed to amplify the 2045-bp fragment at the junction between ISCR1 and the 3 '-CS of the class 1 integrons and used to preliminarily confirm that ISCR1 was inserted downstream from the sul1 gene in the class 1 integrons. Primers I and X, which are specific for the VR1 and VR2 resistance genes investigated in this study, were used to identify the connecting regions: downstream of class 1 integrons and upstream of ISCR1. The amplification products of primers I and X were used as the templates in subsequent PCR analyses. Five set primers (Fig. 1) were used to verify this region with the ISCR 1 and the class 1 integrons connected in series.

Primers of the connection region of integron and ISCRI are listed in Table 2 and were synthesized by Beijing Genomics Institute (Shenzhen, China). PCR amplifications

Table 1 Characterisation of complex class 1 integron in multidrug-resistant Gram-negative bacteria and resistance profiles of sequenced strains

\begin{tabular}{|c|c|c|c|c|}
\hline Species & No. of isolates & Class 1 integron & ISCR & $\begin{array}{l}\text { Type of complex } \\
\text { class } 1 \text { integron }\end{array}$ \\
\hline \multirow[t]{4}{*}{ Escherichia coli } & 2 & $\operatorname{aadB}-a a d A 2$ & anrA1-ampR & B \\
\hline & 1 & dfrA14-arr-2-bla OXA-10-aadA1 & $b / a_{\mathrm{DHA}-1}-a m p R$ & C \\
\hline & 1 & dfrA25 & sapA-like-qnrB2 & $\mathrm{D}$ \\
\hline & 1 & aacA4-arr-3-dfrA27- aadA16 & short chain dehydrogenase/reductase- anrB6 & G \\
\hline \multirow[t]{4}{*}{ Enterobacter cloacae } & 2 & $\operatorname{aadB}-a a d A 2$ & bla & A \\
\hline & 5 & $\operatorname{aadB}-a a d A 2$ & qnrA1- ampR & B \\
\hline & 1 & aacA4-arr-3-dfrA27- aadA16 & short chain dehydrogenase/reductase- anrB6 & G \\
\hline & 1 & aacA4-bla ${ }_{\mathrm{OXA}-101}-c a t \mathrm{~B} 3-a r r-3$ & qnrA1- $a m p R$ & K \\
\hline Enterobacter aerogenes & 2 & aacA4-arr-3-dfrA27-aadA16 & short chain dehydrogenase/reductase- qnrB6 & G \\
\hline \multirow[t]{5}{*}{ Klebsiella pneumoniae } & 1 & $\operatorname{aadB}-a a d A 2$ & qnrA1- ampR & B \\
\hline & 2 & dfrA25 & sapA-like-qnrB2 & $\mathrm{D}$ \\
\hline & 5 & dfrA12-orfF-aadA2 & sapA-like-qnrB2 & $\mathrm{E}$ \\
\hline & 9 & aacA4-arr-3-dfrA27-aadA16 & sapA-like-qnrB2 & $\mathrm{F}$ \\
\hline & 15 & aacA4-arr-3-dfrA27- aadA16 & short chain dehydrogenase/reductase- anrB6 & G \\
\hline Klebsiella oxytoca & 1 & aacA4-arr-3-dfrA27- aadA16 & short chain dehydrogenase/reductase- anrB6 & G \\
\hline Proteus mirabilis & 1 & bla $a_{\text {PSE-1 }}$ & dfrA10 & $\mathrm{L}$ \\
\hline \multirow[t]{3}{*}{ Acinetobacter spp. } & 5 & $\operatorname{aadB}-a a d A 2$ & anrA1-ampR & $\mathrm{B}$ \\
\hline & 3 & catB3-qnrVC-like-aacA4 & bla $a_{P E R-1}-G S T$-novel type $A B C$ transporter & $\mathrm{H}$ \\
\hline & 1 & catB-like-aadB-aadA24-like & bla $_{\text {PER-1 }}$-GST-novel type ABC transporter & J \\
\hline \multirow[t]{2}{*}{ Pseudomonas. aeruginosa } & 2 & $\operatorname{aadB}-a a d A 2$ & qnrA1- ampR & B \\
\hline & 1 & aacA4-like-bla $a_{\mathrm{OXA}-101}-a a d \mathrm{~A} 5$ & bla $_{\text {PER-1 }}$-GST-novel type ABC transporter & । \\
\hline Stenotrophomonas. maltophilia & 1 & $\operatorname{aadB}-a a d A 2$ & anrA1- ampR & $\mathrm{B}$ \\
\hline
\end{tabular}




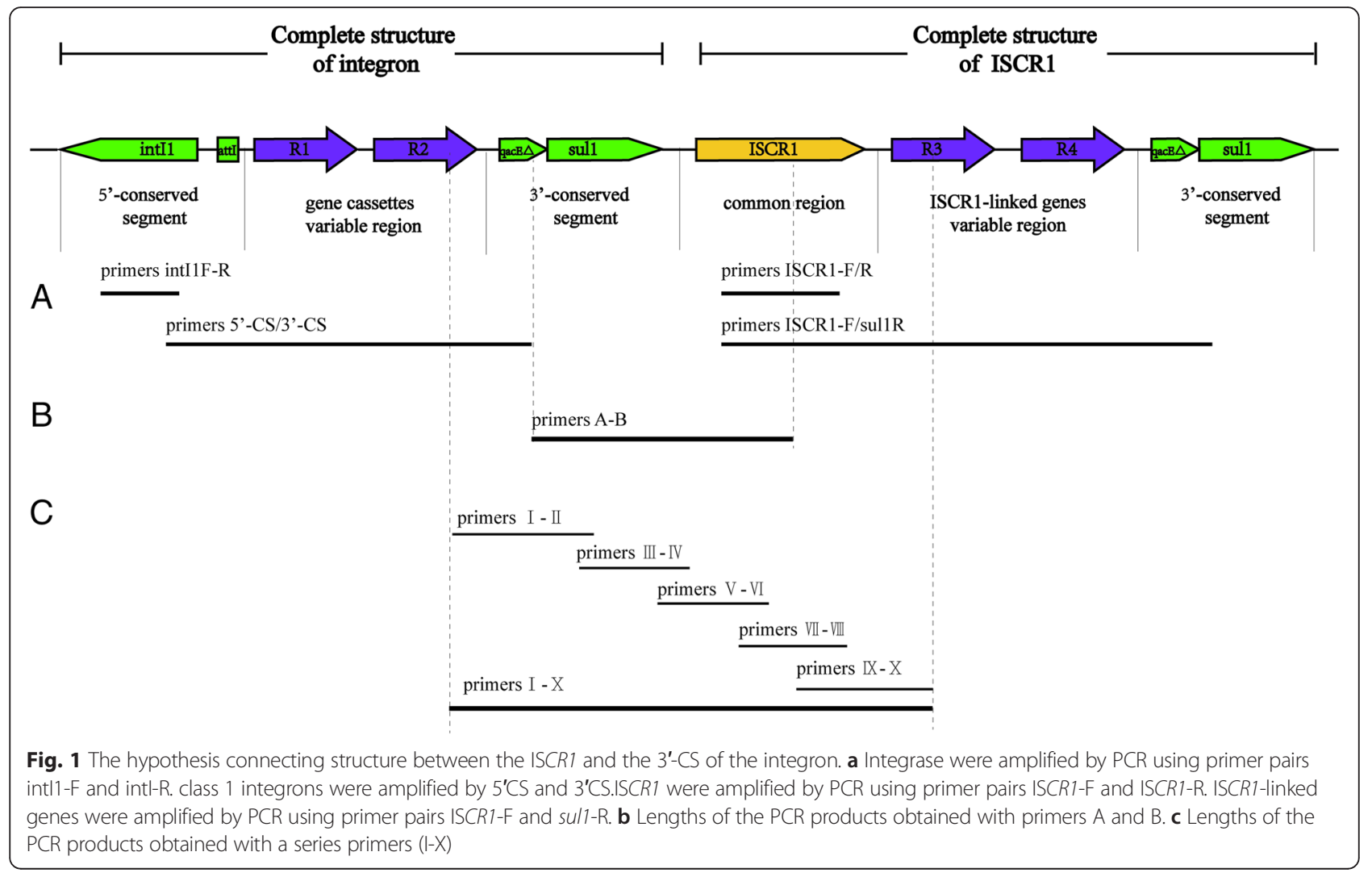

were performed using $1.5 \mu \mathrm{L}$ of template, $2 \mu \mathrm{L}$ of $10 \times$ PCR buffer, $4 \mu \mathrm{M}$ of each primer stock solution, $4 \mathrm{mM}$ of each dNTP, 1 U of Ex Taq DNA polymerase (TaKaRa Bio Inc., Tokyo, Japan), and sterile distilled water added to a final total volume of $20 \mu \mathrm{L}$. Amplification was performed using a Mastercycler PCR System (Eppendorf International, Hamburg, Germany). Thermocycling parameters were $94{ }^{\circ} \mathrm{C}$ for $5 \mathrm{~min}, 35$ cycles of $94{ }^{\circ} \mathrm{C}$ for $30 \mathrm{~s}, 55-60{ }^{\circ} \mathrm{C}$ for $5 \mathrm{~min}$, and $72{ }^{\circ} \mathrm{C}$ for $2-5 \mathrm{~min}$; a final extension step at $72{ }^{\circ} \mathrm{C}$ was added for $5 \mathrm{~min}$. Different annealing temperature and extension of time depended on different length of the PCR amplicons.

The PCR amplicons were purified and sequenced (Sanger capillary sequencing) at the Beijing Genomics Institute (Shenzhen, China). The resulting DNA sequences were analysed with the BLAST program at the NCBI homepage (http://www.ncbi.nlm.nih.gov/blast/).

\section{Results}

Thirteen genes (qnrA1, qnrB2, qnrB6, ampR, dfrA10, $b l a_{\mathrm{DHA}-1}, b l a_{\mathrm{CTX}-\mathrm{M}-9}, b l a_{\mathrm{PER}-1}$, ins $B, \operatorname{sapA-like}$, gst, and those encoding a novel ABC transporter and a shortchain dehydrogenase/reductase) were detected in the ISCR1-linked resistance gene arrays. The gene cassettes of the class 1 integrons found in the 63 isolates included those encoding resistance to trimethoprim (dfrA12, dfrA25, dfrA27), aminoglycosides (aadA2,
aadA16, aadB, aac(6')-Ib, aacA4), chloramphenicol (catB3, catB8), quinolone (qnrVC-like), and rifampicin (arr-3, arr-2).

In this study, the structures (the variable regions of the class 1 integron structures and the ISCR1-linked resistance genes) are connected in series among 63 isolates. We found 12 distinct structures connecting the ISCR 1 and the class 1 integron, with a different genecassette variable regions, composed of the $5^{\prime}$-CS and the 3'-CS but displaying another unique variable region located between ISCR1 and the second copy of the 3'-CS (Fig. 2). The PCR products amplified from the regions connecting the first $3{ }^{\prime}$-CS and ISCR1 are shown in Fig. 3.

\section{Discussion}

To the best of our knowledge, this is the first study to investigate the structure connecting the ISCR 1 and the $3^{\prime}$-CS of the integron in different species in clinical isolates of MDR Gram-negative bacteria on a large scale. It was found that 63 isolates simultaneously carried class 1 integrons and ISCR1. The variable regions of the class 1 integron structures to the ISCR1-linked resistance genes were linked successfully using long-extension PCR, suggesting that these two structures are connected in series.

Of the 12 type structures, 8 types (A, C, F, H, I, J, K and $\mathrm{L}$ type) were first found in any species according to 
Table 2 Primers for PCR amplification of the connection region of integron and ISCR1

\begin{tabular}{|c|c|c|c|}
\hline $\begin{array}{l}\text { Type of complex } \\
\text { class } 1 \text { integron }\end{array}$ & No. & Primer name & Sequence $\left(5^{\prime}-3^{\prime}\right)$ \\
\hline \multirow[t]{4}{*}{$\bar{A}$} & 1 & $a a d A 2+b l a_{C T X-9} F$ & CGTTGCCTTGGTAGGTCC \\
\hline & $\|$ & aadA2 + bla $a_{\text {CTX-9 }}$ up $R$ & TTGCTTGCCATAGTCATCTT \\
\hline & IX & aadA2 $+b l a_{C T X-9}$ down $F$ & CCCCAAGGAGCCCATTC \\
\hline & $x$ & $\operatorname{aadA} 2+b a_{\text {CTX-9 }} R$ & GGTATTCAGCGTAGGTTCAGT \\
\hline \multirow[t]{4}{*}{ B } & 1 & $\operatorname{aadA} 2+\operatorname{anr} \mathrm{A} 1 \mathrm{~F}$ & GTTGTCCCGCATTTGGT \\
\hline & $\|$ & $\operatorname{aadA} 2+\operatorname{anrA} 1$ up $R$ & GGTTGAGCGAGAAGGTITI \\
\hline & IX & aadA2 + anrA1 down F & GCGTGAGCTGCCACCAGAA \\
\hline & $x$ & $\operatorname{aadA} 2+\operatorname{anr} \mathrm{A} 1 \mathrm{R}$ & TCTTATGGCTGACTTGATTGTAG \\
\hline \multirow[t]{4}{*}{ C } & 1 & $a a d A 1+b / a_{\mathrm{DHA}-1} \mathrm{~F}$ & ATCTGGCTATCTTGCTGAC \\
\hline & $\|$ & $\operatorname{aadA} 1+b a_{\mathrm{DHA}-1}$ up $\mathrm{R}$ & TTCCGAGAAGGTGATTGC \\
\hline & IX & $a a d A 1+b l a_{D H A-1}$ down $F$ & CCAACACTGCTCAACACT \\
\hline & $x$ & $a a d A 1+b l a_{\mathrm{DHA}-1} \mathrm{R}$ & GGTGGCGATTGTGATTCT \\
\hline \multirow[t]{4}{*}{ D } & । & dfrA25+ sapA F & ACGAAGCGATGGGTAGA \\
\hline & $\|$ & dfrA25+ sapA up $R$ & AGCCCTCACGAGTTGTTAT \\
\hline & IX & dfrA25+ sapA down $\mathrm{F}$ & CAAGAAGCCCGACAAAT \\
\hline & $x$ & dfrA25+ sapA R & TGGGAGGTGCTGGATAA \\
\hline \multirow[t]{4}{*}{$\mathrm{E}$} & 1 & $\operatorname{aadA} 2+\operatorname{sap} A F$ & CGTTGCCTTGGTAGGTC \\
\hline & $\|$ & $\operatorname{aadA} 2+\operatorname{sap} A$ up $R$ & AACCGCACAATCTCGTC \\
\hline & IX & $\operatorname{aadA} 2+\operatorname{sap} A$ down $F$ & CGCTGCTGATAGACGAAG \\
\hline & $x$ & $\operatorname{aadA} 2+\operatorname{sapA} R$ & TGGGAGGTGCTGGATAA \\
\hline \multirow[t]{4}{*}{$\mathrm{F}$} & I & $\operatorname{aadA} 16+\operatorname{sapA} F$ & GTTGTTCCTTGGCGTTATC \\
\hline & $\|$ & aadA16+ sapA up $\mathrm{R}$ & TCAGCAATATCGGGATAGAG \\
\hline & IX & aadA16+ sapA down $\mathrm{F}$ & AGACGATACGCTGACTCA \\
\hline & $x$ & $\operatorname{aadA} 16+\operatorname{sapA} R$ & ATGACCGACTGCTTGATG \\
\hline \multirow[t]{4}{*}{ G } & 1 & dfrA27 + short chain F & GCAATGAGGGAGCTAAAGA \\
\hline & $\|$ & dfrA27 + short chain up R & TTGGGTTCAGGGTGCTAT \\
\hline & IX & dfrA27 + short chain down F & CAAGAAGCCCGACAAATC \\
\hline & $x$ & dfrA27 + short chain $\mathrm{R}$ & TTCACGAGCATAGGCAATA \\
\hline \multirow[t]{4}{*}{$\mathrm{H}$} & 1 & $a a c A 4+b l a_{P E R} F$ & CCCGAGGTCACCAAGA \\
\hline & $\|$ & $a a c A 4+b l a_{P E R} U P R$ & GCACCATCCCACATAAGA \\
\hline & IX & aacA4 + blaperdown F & AAGAGGGCGAAGACGA \\
\hline & $x$ & $a a c A 4+b l a_{P E R} R$ & TCCATCAGGCAACAGAAT \\
\hline \multirow[t]{4}{*}{1} & । & aadA5 + bla $a_{\text {PER }} F$ & ACTGGTCTCATTGCTCCTA \\
\hline & $\|$ & aadA5 + bla $a_{\text {PER }}$ up $R$ & CGAAGAACCGCACAATCT \\
\hline & IX & aadA5 + bla $a_{\text {PER }}$ down $F$ & CAACACTGCTCAACACTG \\
\hline & $x$ & aadA5 + bla $a_{P E R} R$ & ATTGGTTCGGCTTGACTC \\
\hline \multirow[t]{4}{*}{ J } & I & $a a d A 24+b l a_{P E R} F$ & CATCATTCCGTGGCGTTA \\
\hline & $\|$ & aadA24 + blapER up R & GACACCGAGACCAATAGC \\
\hline & IX & aadA24 + bla $a_{\text {PER }}$ down F & AATCCAACACTGCTCAACA \\
\hline & $x$ & $a a d A 24+b / a_{P E R} R$ & CATCATTCCGTGGCGTTA \\
\hline \multirow[t]{3}{*}{ K } & 1 & $\operatorname{arr}-3+\operatorname{anrA} 1 \mathrm{~F}$ & GGTAATCCAACACAGTCCTA \\
\hline & $\|$ & $\operatorname{arr}-3+\operatorname{anrA1}$ up $\mathrm{R}$ & GTCCGCCTCAGCAATATC \\
\hline & IX & $\operatorname{arr}-3+$ anrA1 down $\mathrm{F}$ & TCCAACACTGCTCAACAC \\
\hline
\end{tabular}


Table 2 Primers for PCR amplification of the connection region of integron and ISCR1 (Continued)

\begin{tabular}{|c|c|c|c|}
\hline & $x$ & $\operatorname{arr}-3+\operatorname{anrA1} \mathrm{R}$ & CCAGAGTATCCGCAATCC \\
\hline \multirow[t]{4}{*}{ L } & । & bla $a_{\mathrm{PSE}-1}+$ drrA10 F & TTATGGCGGCGTTAGATG \\
\hline & $\|$ & $b l a_{\mathrm{PSE}-1}+d f r A 10$ up $\mathrm{R}$ & CGAGACCAATAGCGGAAG \\
\hline & IX & $b a_{\mathrm{PSE}-1}+d f r A 10$ down $\mathrm{F}$ & ATATTGAAGTCTGCGAACAC \\
\hline & $x$ & bla $\mathrm{PSE}-1_{1}+d f \mathrm{fA} 10 \mathrm{R}$ & CGTGCTCTGTGATAGTTGA \\
\hline \multirow[t]{8}{*}{ - } & III & common $1791 \mathrm{~F}$ & TATTGCTGAGGCGGACTG \\
\hline & IV & common $1791 \mathrm{R}$ & CATTGGAGGAGGTCGTTG \\
\hline & V & common $21054 \mathrm{~F}$ & GGCTTCCGCTATTGGTC \\
\hline & $\mathrm{Vl}$ & common $21054 \mathrm{R}$ & TTGCTTGCCATAGTCATCTT \\
\hline & VII & common $31727 \mathrm{~F}$ & TCGCCCACTCAAACAAA \\
\hline & VIII & common $31727 \mathrm{R}$ & GCTCCTCATCCGAAGTATCT \\
\hline & A & - & CCTGTCGGTGTTGCTTAT \\
\hline & B & - & GTTGCTTGCCATAGTCATC \\
\hline
\end{tabular}

Types of complex class 1 integron correspond to Fig. 2;

Primers No. correspond to Fig. 1

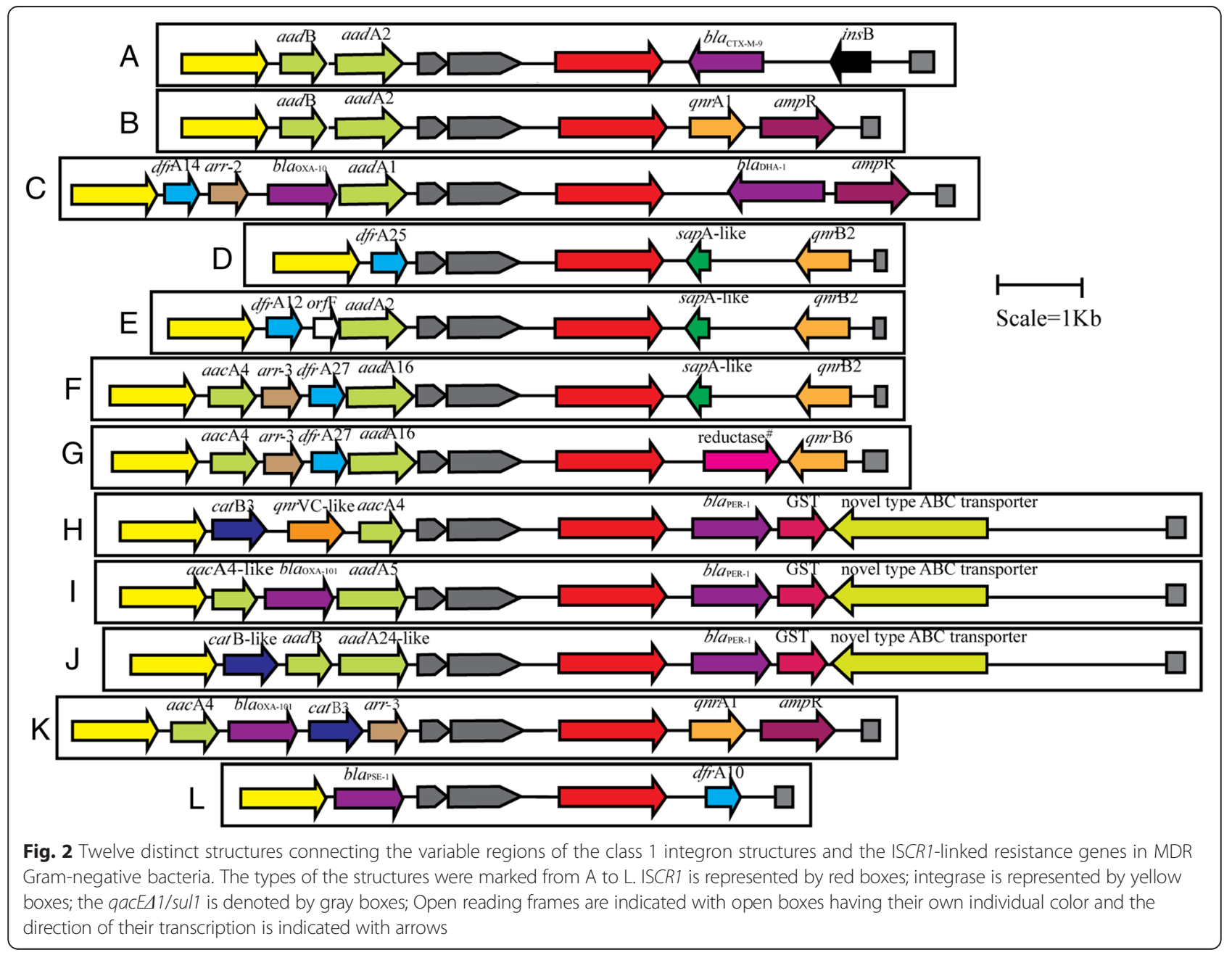




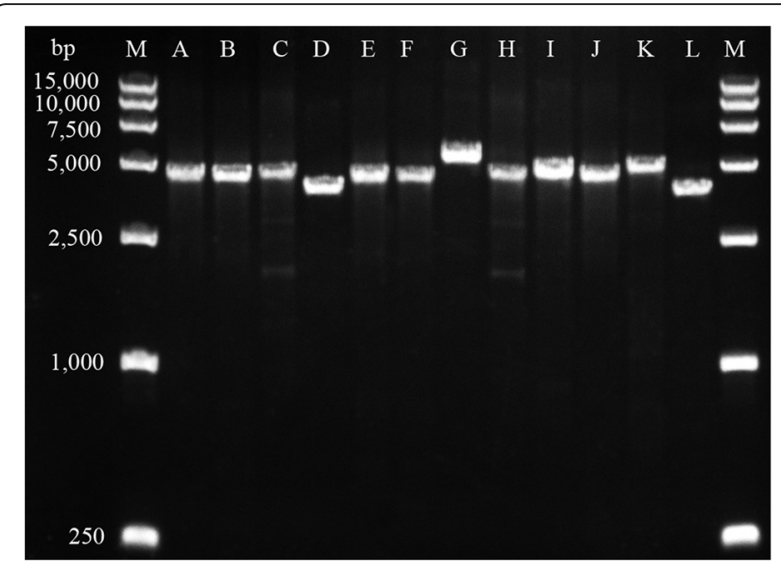

Fig. 3 Agarose gel electrophoresis of PCR products obtained with primers $I$ and $X$ for 12 isolates. primers $I$ and $X$ and the region correspond to Fig. 1. The following types corresponding to the Fig. 2 were showed by lane: 1, marker; 2 A; 3, B; 4, C; 5, D; 6, E; 7, F; 8, G; 9, $\mathrm{H} ; 10, \mathrm{l} ; 11, \mathrm{~J} ; 12, \mathrm{~K} ; 13, \mathrm{~L} ; 14$, marker

the systematic search in PubMed and GenBank. The structure type $\mathrm{K}$ is similar to a structure In37::ISCR1::qnrA1 (accession No. AY259086) [8]. A genetic structure (type B), $[\operatorname{aadB}+\operatorname{aadA2}]: \operatorname{ISCR} 1:[q n r A 1+\operatorname{ampR}]$, is the same as a structure already reported In293::ISCR1::qnrA1 in E. cloacae, [9] which was also found in E. coli, K. pneumoniae, Acinetobacter spp., P. aeruginosa and S. maltophilia in this study. We detected the structure D type, [dfrA25]:ISCR1:[sapA-like + qnrB2], that was previously found and described in Salmonella isolates [10], but which has never before been described in $E$. coli or $K$. pneumoniae. The structure $\mathrm{G}$ has been detected in clinical K. pneumoniae (accession No. JF775516) isolates in a previous study [11] but was first detected in E. coli, E. cloacae, E. aerogenes and $K$. oxytoca. Besides, the structure $\mathrm{G}$ was found in about $32 \%$ isolates (20/63) in this study. The structure E type was previously reported by Ziyong Zong et al. in 2010 from China [12] (accession No. NG037697).

Among the 12 type structures, the VR-2 was similar as previous surveys, whereas the VR-1 was very different from previous surveys. Although the array of gene cassettes in the VR-1 can be easily exchanged, the distribution of ISCR1::qnrB2 in our survey revealed the presence of structure D, E and F. Worldwide, different VR-1 arrays in the structures carrying ISCR1::qnrB2 clinical isolates have been reported eg. In2, In27, In54, In73, In207 and In585 [13]. About structure H, I and J, Ruirui Xia et al. described similar structures carrying $b l a_{\mathrm{PER}-1}$ and $q n r V C$-like genes and made an exhaustive study [14].

Enterobacteriaceae strains carrying the structures are becoming more common [15-17]. In this study, nine distinct structures were identified among Enterobacteriaceae strains, including seven distinct structures connecting the
ISCR 1 and the class 1 integron that have never been described in any species. It should be noted that only four distinct structures were identified in MDR nonfermenting isolates. One possible explanation is that chromosomal resistance mechanisms, such as efflux pumps, are more common than laterally transferred genetic resistance factors in these genera in this bacterial population.

\section{Conclusions}

This is the first study to describe the structure connecting the ISCR 1 and the class 1 integron in clinical MDR Gram-negative bacterial isolates in a large-scale study. In total, 12 distinct structures were described. Several types of these structures are similar with the structure of other reports, but not entirely same. This structure is a powerful gene-capturing tool that can mobilize antibioticresistance genes. Therefore, the structural analysis of the structure connecting the ISCR 1 and the class 1 integron could guide treatment strategies and provide directions for future research into the mechanisms of bacterial antibiotic resistance.

\section{Nucleotide sequence accession number}

The nucleotide sequences of the structure $\mathrm{A}$ to $\mathrm{L}$ in this work have been submitted to the GenBank database and assigned accession No. JX880393, JX880383, KM111274, JX880388, KM111278, KM111276, KM111280, JX880386, KM111273, KM111272, KM111271 and KM111275.

\section{Competing interests}

The authors declare that they have no competing interests.

\section{Authors' contributions}

CC and JS carried out PCR experiments, data organization and analysis and contributed to writing and to the interpretation of the results. FZ participated in the sequence alignment and data analysis. WL and QY collected all of bacteria isolates and clinical data and participated experiments. YR contributed to the design of the study and assisted in the drafting of the manuscript. All authors have read and approved the final manuscript.

\section{Acknowledgements}

None to declare.

\section{Funding}

This study was supported by the grant from Guangdong Province Science and Technology Project (No.2013B010404021 and No.2014A010107011) and Guangzhou City Science and Technology Project (No. 201510010167).

Received: 16 October 2015 Accepted: 29 March 2016 Published online: 21 April 2016

\section{References}

1. Toleman MA, Bennett PM, Walsh TR. ISCR elements: novel gene-capturing systems of the 21st century? Microbiol Mol Biol Rev. 2006;70(2):296-316.

2. Wang F, Wu K, Sun J, Wang Q, Chen Q, Yu S, Rui Y. Novel ISCR1-linked resistance genes found in multidrug-resistant Gram-negative bacteria in southern China. Int J Antimicrob Agents. 2012;40(5):404-8.

3. Wu K, Wang F, Sun J, Wang Q, Chen Q, Yu S, Rui Y. Class 1 integron gene cassettes in multidrug-resistant Gram-negative bacteria in southern China. Int J Antimicrob Agents. 2012;40(3):264-7. 
4. Mazel D. Integrons: agents of bacterial evolution. Nat Rev Microbiol. 2006; 4(8):608-20.

5. Toleman MA, Walsh TR. Combinatorial events of insertion sequences and ICE in Gram-negative bacteria. FEMS Microbiol Rev. 2011;35(5):912-35.

6. Bennett PM. Plasmid encoded antibiotic resistance: acquisition and transfer of antibiotic resistance genes in bacteria. Br J Pharmacol. 2008;153 Suppl 1: S347-357.

7. Partridge SR, Hall RM. In34, a Complex In5 Family Class 1 Integron Containing orf513 and dfrA10. Antimicrob Agents Chemother. 2003;47(1):342-9.

8. Wang M, Tran JH, Jacoby GA, Zhang Y, Wang F, Hooper DC. Plasmidmediated quinolone resistance in clinical isolates of Escherichia coli from Shanghai, China. Antimicrob Agents Chemother. 2003;47(7):2242-8.

9. Bado I, Cordeiro NF, Robino L, Garcia-Fulgueiras V, Seija V, Bazet C, Gutkind G, Ayala JA, Vignoli R. Detection of class 1 and 2 integrons, extendedspectrum beta-lactamases and gnr alleles in enterobacterial isolates from the digestive tract of Intensive Care Unit inpatients. Int J Antimicrob Agents. 2010;36(5):453-8.

10. Wu JJ, Ko WC, Chiou CS, Chen HM, Wang LR, Yan JJ. Emergence of Qnr determinants in human Salmonella isolates in Taiwan. J Antimicrob Chemother. 2008;62(6):1269-72.

11. Ruiz E, Saenz Y, Zarazaga M, Rocha-Gracia R, Martinez-Martinez L, Arlet G, Torres C. gnr, aac(6)--lb-cr and gepA genes in Escherichia coli and Klebsiella spp.: genetic environments and plasmid and chromosomal location. J Antimicrob Chemother. 2012;67(4):886-97.

12. Zong Z, Partridge SR, Iredell JR. ISEcp 1-mediated transposition and homologous recombination can explain the context of bla(CTX-M-62) linked to gnrB2. Antimicrob Agents Chemother. 2010;54(7):3039-42.

13. Quiroga MP, Arduino SM, Merkier AK, Quiroga C, Petroni A, Argentinian Integron Study G, Roy PH, Centron D. "Distribution and functional identification of complex class 1 integrons". Infect Genet Evol. 2013;19:88-96.

14. Xia R, Guo X, Zhang Y, Xu H. gnrVC-like gene located in a novel complex class 1 integron harboring the ISCR1 element in an Aeromonas punctata strain from an aquatic environment in Shandong Province, China. Antimicrob Agents Chemother. 2010;54(8):3471-4.

15. Richter SN, Frasson I, Bergo C, Manganelli R, Cavallaro A, Palu G. Characterisation of qnr plasmid-mediated quinolone resistance in Enterobacteriaceae from Italy: association of the gnrB19 allele with the integron element ISCR1 in Escherichia coli. Int J Antimicrob Agents. 2010; 35(6):578-83.

16. Santos C, Caetano T, Ferreira S, Ramalheira E, Mendo S. A novel complex class 1 integron found in a Klebsiella pneumoniae isolate from Portugal. Clin Microbiol Infect. 2011;17(7):1036-9.

17. Sun C, Su Z, Zhou C, Liu Y, Yuan H, Yin J, Xu H. Complex class 1 integron containing bla (CTX-M-1) genes isolated from Escherichia coli: a potentially novel resistant gene-capturing tool kit. Curr Microbiol. 2012;64(3):265-70

\section{Submit your next manuscript to BioMed Central and we will help you at every step:}

- We accept pre-submission inquiries

- Our selector tool helps you to find the most relevant journal

- We provide round the clock customer support

- Convenient online submission

- Thorough peer review

- Inclusion in PubMed and all major indexing services

- Maximum visibility for your research

Submit your manuscript at www biomedcentral.com/submit

) Biomed Central 